\title{
Design and characterization of electrons in a fractal geometry
}

\author{
S. N. Kempkes ${ }^{\# 1}$, M.R. Slot ${ }^{\# 2}$, S. E. Freeney², S. J. M. Zevenhuizen², D. Vanmaekelbergh², I. \\ Swart $^{2, \dagger}$, and C. Morais Smith ${ }^{1, \dagger}$
}

${ }^{1}$ Institute for Theoretical Physics, Utrecht University, Netherlands ${ }^{2}$ Debye Institute for Nanomaterials Science, Utrecht University, Netherlands

\# These authors contributed equally to this work.

\section{Abstract}

The dimensionality of an electronic quantum system is decisive for its properties. In one dimension electrons form a Luttinger liquid and in two dimensions they exhibit the quantum Hall effect. However, very little is known about the behavior of electrons in non-integer, or fractional dimensions1. Here, we show how arrays of artificial atoms can be defined by controlled positioning of $\mathrm{CO}$ molecules on a $\mathrm{Cu}$ (111) surface2-4, and how these sites couple to form electronic Sierpiński fractals. We characterize the electron wave functions at different energies with scanning tunneling microscopy and spectroscopy and show that they inherit the fractional dimension. Wave functions delocalized over the Sierpiński structure decompose into self-similar parts at higher energy, and this scale invariance can also be retrieved in reciprocal space. Our results show that electronic quantum fractals can be artificially created by atomic manipulation in a scanning tunneling microscope. The same methodology will allow future study to address fundamental questions about the effects of spin-orbit interaction and a magnetic field on electrons in non-integer dimensions. Moreover, the rational concept of artificial atoms can readily be transferred to planar semiconductor electronics, allowing for the exploration of electrons in a welldefined fractal geometry, including interactions and external fields.

Fractals have been investigated in a wide variety of research areas, ranging from polymers5, porous systems6, electrical storage7 and stretchable electronics8 down to molecular5,9-11 and plasmonic12 fractals. On the quantum level, fractal properties emerge in the behavior of electrons under perpendicular magnetic fields, for example in the Hofstadter butterfly 13 and in quantum Hall resistivity 14,15. In addition, a multi-fractal behavior has been observed for

Users may view, print, copy, and download text and data-mine the content in such documents, for the purposes of academic research, subject always to the full Conditions of use:http://www.nature.com/authors/editorial_policies/license.html\#terms

${ }^{\dagger}$ Correspondence and requests for materials should be addressed to C.M.S. (c.demoraissmith@uu.nl) and I.S. (i.swart@uu.nl).

Data availability

All data is available from the corresponding authors on reasonable request. The experimental data can be accessed using open-source tools.

Author contributions S.N.K. did the calculations under the supervision of C.M.S. The experiments were performed by M.R.S. with contributions from S.E.F. and S.J.M.Z. under the supervision of I.S. and D.V. All authors contributed to the interpretation of the data and to the manuscript.

Author information Reprints and permissions information is available at www.nature.com/reprints.

Authors declare no competing interest. 
the wave functions at the transition from a localized to delocalized regime in disordered electronic systems 16-18. However, these systems do not allow one to study the influence of non-integer dimensions on the electronic properties. Geometric electronic fractals, in which electrons are confined to a self-similar fractal geometry with a dimension between one and two, have only been studied from a theoretical perspective. For these fractals, a recurrent pattern in the density of states as well as extended and localized electronic states were predicted19-22. Recently, simulations of quantum transport in fractals revealed that the conductance fluctuations are related to the fractal dimension23, and that the conductance in a Sierpiński fractal shows scale-invariant properties24-26.

Here, we report how to construct and characterize, in a controlled fashion, a fractal lattice with electrons: the electrons that reside on a $\mathrm{Cu}(111)$ surface are confined to a self-similar Sierpiński geometry through atomic manipulation of $\mathrm{CO}$ molecules on the $\mathrm{Cu}(111)$ surface. Manipulation of surface-state electrons by adsorbates has been pioneered by Crommie et al. 27 and has been used to create electronic lattices "on demand" such as a molecular graphene2, an electronic Lieb lattice3,28, a checkerboard and stripe-shaped lattice29, and a quasiperiodic Penrose tiling4. We characterized the first three generations of an electronic Sierpiński triangle by scanning tunneling microscopy and spectroscopy, acquiring the spatially and energy-resolved electronic local density of states (LDOS). These results were corroborated by muffin-tin calculations as well as tight-binding simulations based on artificial atomic $s$-orbitals coupled in the Sierpiński geometry.

The Sierpiński triangle with Hausdorff dimension $\log (3) / \log (2)=1.58$ is presented in Fig. 1a. We define atomic sites at the corners and in the center of the light blue triangles as shown in Fig. 1b for the first generation $G(1) 10,30$. $G(1)$ has three inequivalent atomic sites, indicated in red, green and blue, which differ by their connectivity. A triangle of generation $G(N)$ consists of three triangles $G(N-1)$, sharing the red corner sites. The surface-state electrons of $\mathrm{Cu}(111)$ are confined to the atomic sites by adsorbed $\mathrm{CO}$ molecules, acting as repulsive scatterers. Fig. 1c shows the experimental realization of the first three generations of the Sierpiński triangle and Fig. 1d shows the relation with the artificial atomic sites. The distance between neighboring sites is $1.1 \mathrm{~nm}$, such that the electronic structure of the fractal will emerge in an experimentally suitable energy range 2.

Figure 1e presents the experimental LDOS at the red, blue and green atomic sites in the $G(3)$ Sierpiński triangle (indicated by the open circles in Fig. 1c). The differential conductance $(d I / d V)$ spectra were normalized by the average spectrum taken on the bare $\mathrm{Cu}(111)$ surface, similar to Ref. 2. The onset of the surface state band is located at $V=-0.45 \mathrm{~V}$. We focus on the bias window between $-0.4 \mathrm{~V}$ and $0.3 \mathrm{~V}$. Around $V=-0.3 \mathrm{~V}$ the LDOS on the red, green and blue sites is nearly equal, whereas slightly above $V=-0.2 \mathrm{~V}$, the red sites exhibit a remarked minimum, while the green and blue sites show a considerably higher LDOS. At $V$ $=-0.1 \mathrm{~V}$, the blue sites show a minimum, while the red and green sites exhibit a pronounced maximum in the LDOS. At $V=+0.1 \mathrm{~V}$, the blue sites show a larger peak in the differential conductance, while the green and red sites exhibit a smaller peak. The experimental LDOS is in good agreement with both the tight-binding (see Fig. 1f) and muffin-tin simulations (see Supplementary Information). This finding corroborates that our design leads to the desired confinement of the 2D electron gas to the atomic sites of the Sierpiński geometry. In 
addition, it allows us to characterize the wave functions of the chosen Sierpiński geometry in detail.

Figure 2 shows experimental wave-function maps obtained at different bias voltages and a comparison with simulations using a tight-binding and muffin-tin model. In a thought experiment, we will discuss how electrons can be transported across the setup between a source and a drain at arbitrary positions. At a bias voltage of $-0.325 \mathrm{~V}$, the red (R), green (G) and blue (B) sites all have a high LDOS, and this also holds between the sites. Hence, from a chemical perspective, this wave function has strong bonding character, yielding an excellent conductivity from source to drain along (R-B-G-B-R)-pathways. At $V=-0.2 \mathrm{~V}$, the red sites that connect the $G(1)$ triangles have a low amplitude: the wave function of the $G(3)$ triangle partitions into 9 parts, each corresponding to a $G(1)$ triangle. The self-similar Sierpiński geometry thus leads to a subdivision of a fully bonding wave function delocalized over the $G(3)$ Sierpiński triangle at $-0.325 \mathrm{~V}$ in self-similar $G(1)$ parts at $-0.2 \mathrm{~V}$, demonstrating self-similar properties of the LDOS itself. At the latter bias voltage, the conductivity along (R-B-G-B-R)-pathways suffers from the lower amplitude on the red sites (except the red corner sites). At $V=-0.1 \mathrm{~V}$, the LDOS shows a marked minimum on the blue sites and a peak at the green and red sites. From the tight-binding calculation, we find that the wave function has nodes on the blue sites, corresponding to a non-bonding molecular orbital from a chemical perspective. It is clear that the conductivity along the (RB-G-B-R)-pathway mediated by nearest-neighbor hopping has vanished, and that electrons have to perform next-nearest-neighbor hopping between the red and green sites to propagate. These results connect with the theoretically calculated transmission of a Sierpiński carpet on a hexagonal lattice, which exhibits a gap in the conductivity although there is a high DOS in the system23. Finally, at $V=+0.1 \mathrm{~V}$, all blue sites in the $G(3)$ Sierpiński structure have a high amplitude, whereas the red and green sites exhibit a low amplitude. Again, the conductivity between source and drain is suppressed. We note that the LDOS maps of the three generations $G(1)-G(3)$ show the same features (see Supplementary Information), which is a consequence of the self-similarity of the geometry. We study this scale-invariance of the wave function in more detail with the box-counting method.

In order to determine whether the electronic wave functions inside the Sierpiński structure inherit the scaling properties of the Sierpiński geometry, we determine the fractal dimension of the wave-function maps at different energies. We calculate the box-counting dimension (also called Minkowski-Bouligand dimension) for both the experimental and simulated muffin-tin LDOS maps using $D=\lim _{r \rightarrow 0} \frac{\log N(r)}{\log (1 / r)}$, with $N$ the number of circles required to cover the contributing LDOS and $r$ the radius of these circles. In this procedure, the number of circles $N$ is counted for various $r$, and subsequently the fractal dimension is given by the slope of the log-log plot for $N(r)$. The method is presented in Fig. 3a and more details are given in the Supplementary Information. Fig. $3 b$ shows the box-counting dimension obtained experimentally (dark orange) and theoretically (light orange) for the wave-function maps acquired at different energies (see e.g. Fig. 2). For comparison, we also show the dimension obtained from the wave-function maps of a square lattice (dark and light blue, for the experiment and theory respectively), realized in the same way and measured in the same energy window3. The difference between the experimental and simulated maps is ascribed 
to a more gradual contrast in the simulation, where also contributions of the tip density of states do not play a role. Fluctuations in the calculated dimension at higher energy occur due to the nodes that appear in the maps at higher energies. These nodes are not included as a part of the fractal set (LDOS amplitude is below the counting threshold) and therefore the calculated dimension is affected as the energy is increased. It can be clearly seen that the box-counting dimension of the Sierpiński triangle is close to the theoretical Hausdorff dimension 1.58 (orange solid line), while the square lattice has a dimension close to 2 (blue solid line). From these results, we conclude that the wave functions inherit the fractal dimension and therefore the scaling properties of the geometry to which they are confined, and that this dimension can be non-integer.

Finally, we show how the self-similarity of the wave-function maps is reflected in momentum space. The Fourier-transformed wave-function map at $V=-325 \mathrm{mV}$ (Fig. 4a) exhibits remarked maxima at $k=1.9 \mathrm{~nm}^{-1}$ (turquoise), $k=1.0 \mathrm{~nm}^{-1}$ (red), and $k=0.5 \mathrm{~nm}^{-1}$ (yellow). These maxima correspond to the next-nearest-neighbor distances between the artificial atomic sites (see Fig. 1), the side of a $G(1)$ triangle, and the side of a $G(2)$ triangle in real space, respectively. We then transform parts of the Fourier map back into real space (Fig. 4b-d). The data inside the turquoise circle recover the full $G(3)$ Sierpiński triangle, as shown in Fig. 4b. Transforming the values inside the red circle, however, results in a Sierpiński triangle of generation 2, while the size is retained (see Fig. 4c). Analogously, transforming the data inside the yellow circle yields a first-generation Sierpiński triangle (Fig. 4d). This shows that the $G(3)$ wave function contains Fourier terms of the prior generations. The self-similar features of the Sierpiński triangle are thus inherently encoded in momentum space.

We have demonstrated a rational concept of building electronic wave functions with a fractional dimension from artificial atomic sites that couple in a controlled way. We discussed the wave functions that form by coupling the $s$-orbitals of artificial atoms in the single-electron regime. While this study represents the simplest case, it already displays several aspects of fractal confinement. The emergent fractionalisation of the wave function at the single-particle level has profound implications and opens a series of interesting questions for future investigation: Do electrons in $\mathrm{D}=1.58$ behave like Luttinger liquids? Do they exhibit the fractional quantum Hall effect in the presence of a strong perpendicular magnetic field, or is the behaviour hybrid between 1D and 2D? How does charge fractionalisation manifest when the wave function is itself already fractional? Recent theoretical work already addresses parts of these questions and corroborates the potential of electrons in fractal lattices, showing that the Sierpiński carpet and gasket host topologically protected states in the presence of a perpendicular magnetic field31. Furthermore, the design of artificial-atom quantum dots coupled in a fractal geometry can also be implemented in semiconductor technology, thus making it possible to perform spectroscopy and transport experiments under controlled electron density. This would form a versatile platform to explore fractal electronics with several internal degrees of freedom, such as orbital type, Coulomb and spin-orbit interactions, as well as external electric and magnetic fields. 


\section{Methods}

\section{Scanning tunneling microscope experiments}

The scanning tunneling microscopy and spectroscopy experiments were performed in a Scienta Omicron LT-STM system at a temperature of $4.5 \mathrm{~K}$ and a base pressure around $10^{-10}-10^{-9}$ mbar. A clean $\mathrm{Cu}(111)$ crystal, prepared by multiple cycles of $\mathrm{Ar}^{+}$sputtering and annealing, was cooled down in the scanning tunneling microscope head. Carbon monoxide was leaked into the chamber at $p \approx 3 \cdot 10^{-8}$ mbar for three minutes and adsorbed at the cold $\mathrm{Cu}(111)$ surface. A Cu-coated tungsten tip was used for both the assembly and the characterization of the fractal. The CO manipulation was performed in feedback at $I=60$ $\mathrm{nA}$ and $V=50 \mathrm{mV}$, comparable to previously reported values 32,33 , and was partly automated using an in-house developed program. Scanning-tunneling-microscopy imaging was performed in constant-current mode. A standard lock-in amplifier was used to acquire differential conductance spectra $(f=973 \mathrm{~Hz}$, modulation amplitude $5 \mathrm{mV}$ r.m.s.) and maps ( $f$ $=273 \mathrm{~Hz}$, modulation amplitude $10 \mathrm{mV}$ r.m.s.) in constant-height mode. The Fourier analyses were performed using the software Gwyddion.

\section{Tight-binding calculations}

The atomic sites in the first three generations of the Sierpiński triangle34 are modeled as $s$ orbitals, for which electron hopping between nearest-neighbor and next-nearest-neighbor sites is defined. The parameters used are $e_{s}=-0.1 \mathrm{eV}$ for the on-site energy, $t=0.12 \mathrm{eV}$ for the nearest-neighbor hopping and $t^{\prime} / t=0.08$ for the next-nearest-neighbor hopping, similar to the values reported in Ref. 2. Furthermore, we included an overlap integral $s=0.2$ between nearest-neighbors and solved the generalized eigenvalue equation $H|\psi\rangle=E \mathcal{S}|\psi\rangle$, where $\mathcal{S}$ is the overlap-integral matrix. The LDOS is calculated at each specific atomic site and a Lorentzian energy-level broadening of $\Gamma=0.8 \mathrm{eV}$ is included to account for bulk scattering. For the simulation of the LDOS maps, the same energy-level broadening was used and the LDOS at each site was multiplied with a Gaussian wave function of width $\sigma=$ $0.65 a$, where $a=1.1 \mathrm{~nm}$ is the distance between two neighboring sites.

\section{Muffin-tin calculations}

The surface-state electrons of $\mathrm{Cu}(111)$ are considered to form a 2D electron gas confined between the $\mathrm{CO}$ molecules, which are modeled as filled circles with a repulsive potential of $0.9 \mathrm{eV}$ and radius $R=0.55 \mathrm{a} / 2$. The Schrödinger equation is solved for this particular potential landscape, and a Lorentzian broadening of $\Gamma=0.8 \mathrm{eV}$ is used to account for the bulk scattering.

\section{Box-counting method}

The Minkowski-Bouligand35 or box-counting method is a useful tool to determine the fractal dimension of a certain image, but has to be handled with care. In particular, as was shown in Ref. 36, the size of the boxes needs to be chosen within a certain radius. More specifically, the largest box should not be more than $25 \%$ of the entire image and the smallest box is chosen to be the point at which the slope starts to deviate from the linear regime in the $\log (N)$ vs. $\log (1 / r)$ plot. Redundant features such as the background Friedel 
oscillations were removed by applying a mask. Furthermore, the wave-function maps are not binary, and therefore it is necessary to specify the threshold value above which the pixels are part of the fractal set. The threshold is a certain percentage of the maximum amplitude of the wave-function map at a specific energy. The error introduced by the choice of the threshold is accounted for by performing the calculation procedure for several threshold percentages: between $45 \%$ and $65 \%$ for the experimental wave-function maps, and $60 \%, 75 \%$, and $90 \%$ for the top, center and bottom of the error bar for the simulated (muffin-tin) LDOS maps (see Supplementary Information for the differences in the LDOS for these thresholds). The differences in threshold between experiment and simulation arise because the maps from the simulation are more pronounced than the experimental ones. In addition, due to the dependence on the tip, the experimental maps cannot always be directly compared to each other (see Supplementary Information). Another error sets in by determining the slope of the loglogplot and specifying which radii are taken into account. In the Fig. 3b, the error bars therefore show the maximum value of these two independent errors.

\section{Supplementary Material}

Refer to Web version on PubMed Central for supplementary material.

\section{Acknowledgments}

We thank Guido C.P. van Miert for the discussions. We acknowledge funding from NWO via grants 16PR3245 and DDC13, as well as an ERC Advanced Grant 'FIRSTSTEP' 692691.

\section{References}

[1]. Mandelbrot, BB. The Fractal Geometry of Nature. Freeman, WH, editor. 1982.

[2]. Gomes KK, Mar W, Ko W, Guinea F, Manoharan HC. Designer Dirac fermions and topological phases in molecular graphene. Nature. 2012; 483:306-310. [PubMed: 22422264]

[3]. Slot MR, et al. Experimental realization and characterization of an electronic Lieb lattice. Nature Physics. 2017; 13:672-676. [PubMed: 28706560]

[4]. Collins LC, Witte TG, Silverman R, Green DB, Gomes KK. Imaging quasiperiodic electronic states in a synthetic Penrose tiling. Nature Communications. 2017; 8:15961.

[5]. Newkome GR, et al. Nanoassembly of a Fractal Polymer: A Molecular "Sierpinski Hexagonal Gasket". Science. 2006; 312:1782-1785. [PubMed: 16690820]

[6]. Yu B. Analysis of flow in fractal porous media. Applied Mechanics Reviews. 2008; 61:050801.

[7]. Dubal DP, Ayyad O, Ruiz V, Gomez-Romero P. Hybrid energy storage: the merging of battery and supercapacitor chemistries. Chemical Society Reviews. 2015; 44:1777-1790. [PubMed: 25623995]

[8]. Fan JA, et al. Fractal design concepts for stretchable electronics. Nature Communications. 2014; 5

[9]. Rothemund PW, Papadakis N, Winfree E. Algorithmic self-assembly of DNA Sierpinski triangles. PLoS biology. 2004; 2:e424. [PubMed: 15583715]

[10]. Shang J, et al. Assembling molecular Sierpiński triangle fractals. Nature Chemistry. 2015; 7:389_ 393.

[11]. Li C, et al. Construction of Sierpiński Triangles up to the Fifth Order. Journal of the American Chemical Society. 2017; 139:13749-13753. [PubMed: 28885024]

[12]. De Nicola F, et al. Multiband Plasmonic Sierpinski Carpet Fractal Antennas. ACS Photonics. 2018; 5:2418-2425.

[13]. Hofstadter DR. Energy levels and wave functions of Bloch electrons in rational and irrational magnetic fields. Phys Rev B. 1976; 14:2239-2249. 
[14]. Pan W, et al. Fractional Quantum Hall Effect of Composite Fermions. Physical Review Letters. 2003; 90:016801. [PubMed: 12570639]

[15]. Goerbig MO, Lederer P, Smith CM. On the self-similarity in quantum Hall systems. Europhysics Letters. 2004; 68:72-78.

[16]. Morgenstern M, Klijn J, Meyer C, Wiesendanger R. Real-Space Observation of Drift States in a Two-Dimensional Electron System at High Magnetic Fields. Phys Rev Lett. 2003; 90:056804. [PubMed: 12633386]

[17]. Richardella A, et al. Visualizing Critical Correlations Near the Metal-Insulator Transition in $\mathrm{Ga}_{1-}$ ${ }_{X} \mathrm{Mn}_{X}$ As. Science. 2010; 327:665-669. [PubMed: 20133566]

[18]. Evers F, Mirlin AD. Anderson transitions. Rev Mod Phys. 2008; 80:1355-1417.

[19]. Domany E, Alexander S, Bensimon D, Kadanoff LP. Solutions to the Schrödinger equation on some fractal lattices. Phys Rev B. 1983; 28:3110-3123.

[20]. Ghez J, Wang YY, Rammal R, Pannetier B, Bellissard J. Band spectrum for an electron on a Sierpinski gasket in a magnetic field. Solid State Communications. 1987; 64:1291-1294.

[21]. Andrade RFS, Schellnhuber HJ. Exact Treatment of Quantum States on a Fractal. Europhysics Letters. 1989; 10:73-78.

[22]. Wang XR. Localization in fractal spaces: Exact results on the Sierpinski gasket. Phys Rev B. 1995; 51:9310-9313.

[23]. van Veen E, Yuan S, Katsnelson MI, Polini M, Tomadin A. Quantum transport in Sierpinski carpets. Phys Rev B. 2016; 93:115428.

[24]. Chakrabarti A, Bhattacharyya B. Sierpinski gasket in a magnetic field: electron states and transmission characteristics. Phys Rev B. 1997; 56:13768-13773.

[25]. Liu Y, Hou Z, Hui PM, Sritrakool W. Electronic transport properties of Sierpinski lattices. Phys Rev B. 1999; 60:13444-13452.

[26]. Lin Z, Cao Y, Liu Y, Hui PM. Electronic transport properties of Sierpinski lattices in a magnetic field. Phys Rev B. 2002; 66:045311.

[27]. Crommie MF, Lutz CP, Eigler DM. Confinement of Electrons to Quantum Corrals on a Metal Surface. Science. 1993; 262:218-220. [PubMed: 17841867]

[28]. Drost R, Ojanen T, Harju A, Liljeroth P. Topological states in engineered atomic lattices. Nature Physics. 2017; 13:668-671.

[29]. Girovsky J, et al. Emergence of quasiparticle Bloch states in artificial crystals crafted atombyatom. SciPost Phys. 2017; 2:020.

[30]. Oftadeh R, Haghpanah B, Vella D, Boudaoud A, Vaziri A. Optimal Fractal-Like Hierarchical Honeycombs. Phys Rev Lett. 2014; 113:104301. [PubMed: 25238362]

[31]. Brzezińska M, Cook AM, Neupert T. Topology in the Sierpiński-Hofstadter problem. ArXiv eprints. 2018

[32]. Meyer G, et al. Controlled Manipulation of Atoms and Small Molecules with a Low Temperature Scanning Tunneling Microscope. Single Molecules. 2000; 1:79-86.

[33]. Celotta RJ, et al. Invited Article: Autonomous assembly of atomically perfect nanostructures using a scanning tunneling microscope. Review of Scientific Instruments. 2014; 85:121301. [PubMed: 25554264]

[34]. Sierpiński W. Sur une courbe dont tout point est un point de ramification. C R A S. 1915; 160:302-315.

[35]. Bouligand G. Sur la notion d'ordre de mesure d'un ensemble plan. Bull Sci Math. 1929; 2:185192.

[36]. Foroutan-pour K, Dutilleul P, Smith D. Advances in the implementation of the box-counting method of fractal dimension estimation. Applied Mathematics and Computation. 1999; 105:195210. 
a

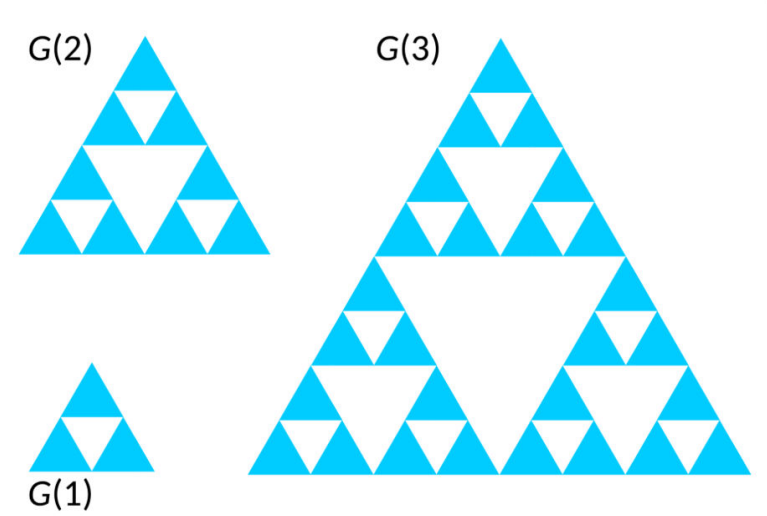

b Tight-binding model

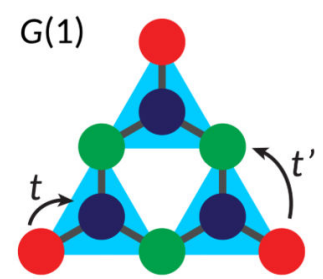

C

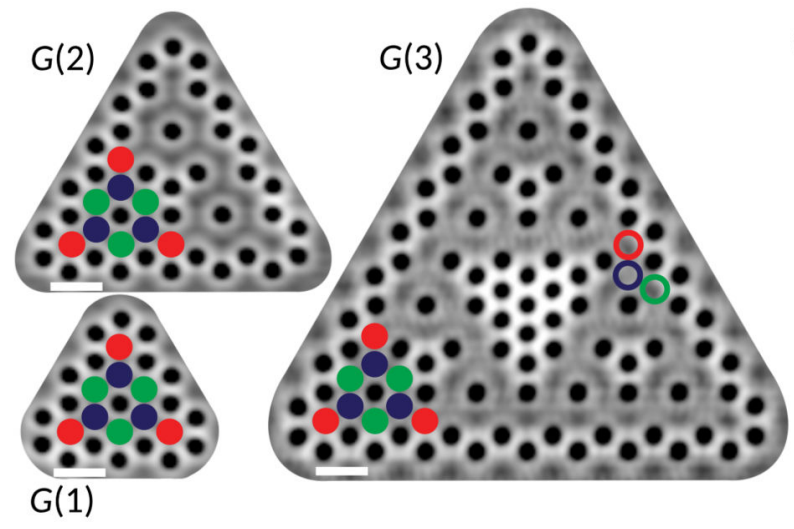

d Muffin-tin

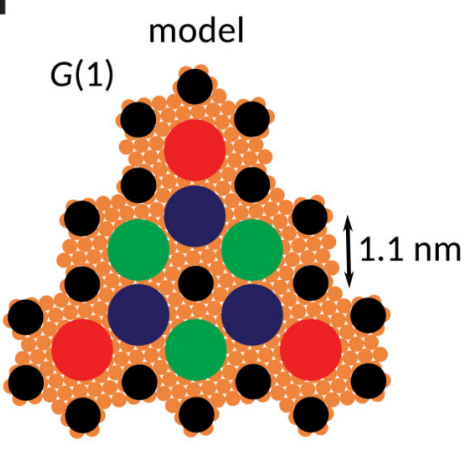

e
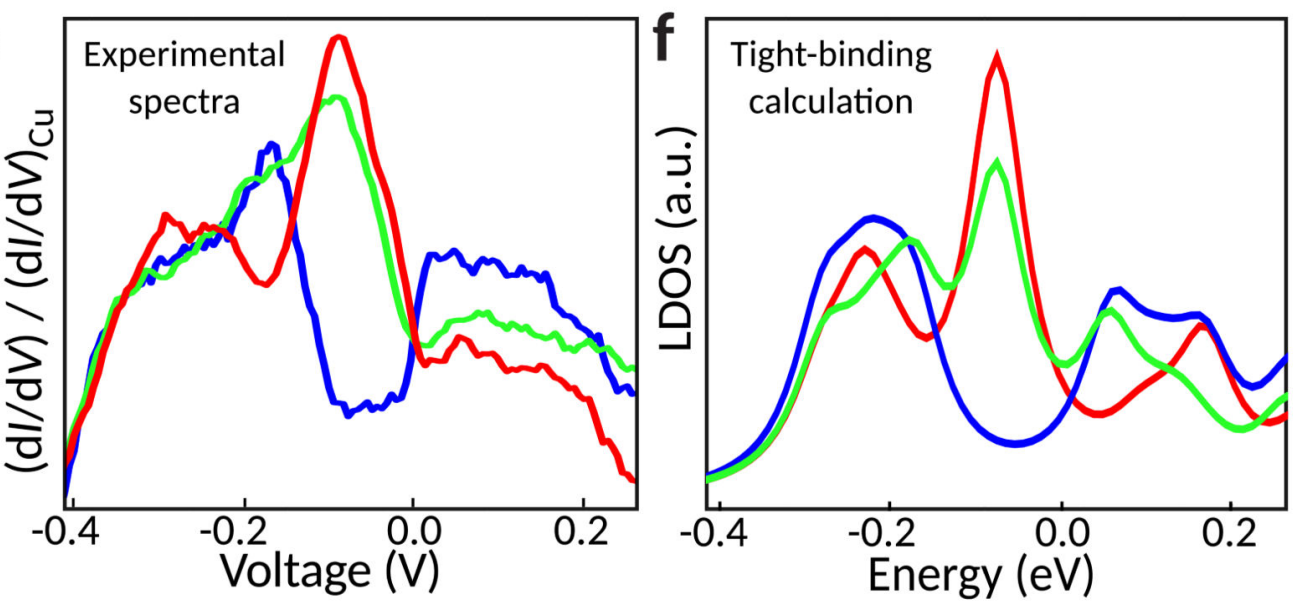

Figure 1. Geometry of the Sierpiński triangle fractal.

a, Schematic of Sierpiński triangles of the first three generations $G(1)-G(3) . G(1)$ is an equilateral triangle subdivided in four identical triangles, from which the center triangle is removed. Three $G(1)(G(2))$ triangles are combined to form a $G(2)(G(3))$ triangle. b, Geometry of a $G(1)$ Sierpiński triangle with red, green and blue atomic sites. $t$ and $t^{\prime}$ indicate nearest-neighbor and next-nearest-neighbor hopping between the sites in the tightbinding model. c, Constant-current STM images of the realized $G(1)-G(3)$ Sierpiński triangles. The atomic sites of one $\mathrm{G}(1)$ building block are indicated as a guide to the eye. 
Imaging parameters: $I=1 \mathrm{nA}, V=1 \mathrm{~V}$ for $G(1)-G(2)$ and $0.30 \mathrm{~V}$ for $G(3)$. Scale bar, 2 $\mathrm{nm}$. $\mathbf{d}$, The configuration of $\mathrm{CO}$ molecules (black) on $\mathrm{Cu}(111)$ to confine the surface-state electrons to the atomic sites of the Sierpiński triangle. e, Normalized differential conductance spectra acquired above the positions of red, blue and green open circles in $\mathbf{c}$ (and equivalent positions). f, LDOS at the same positions, simulated using a tight-binding model with $t=0.12 \mathrm{eV}, t^{\prime}=0.01 \mathrm{eV}$ and an overlap $s=0.2$. 


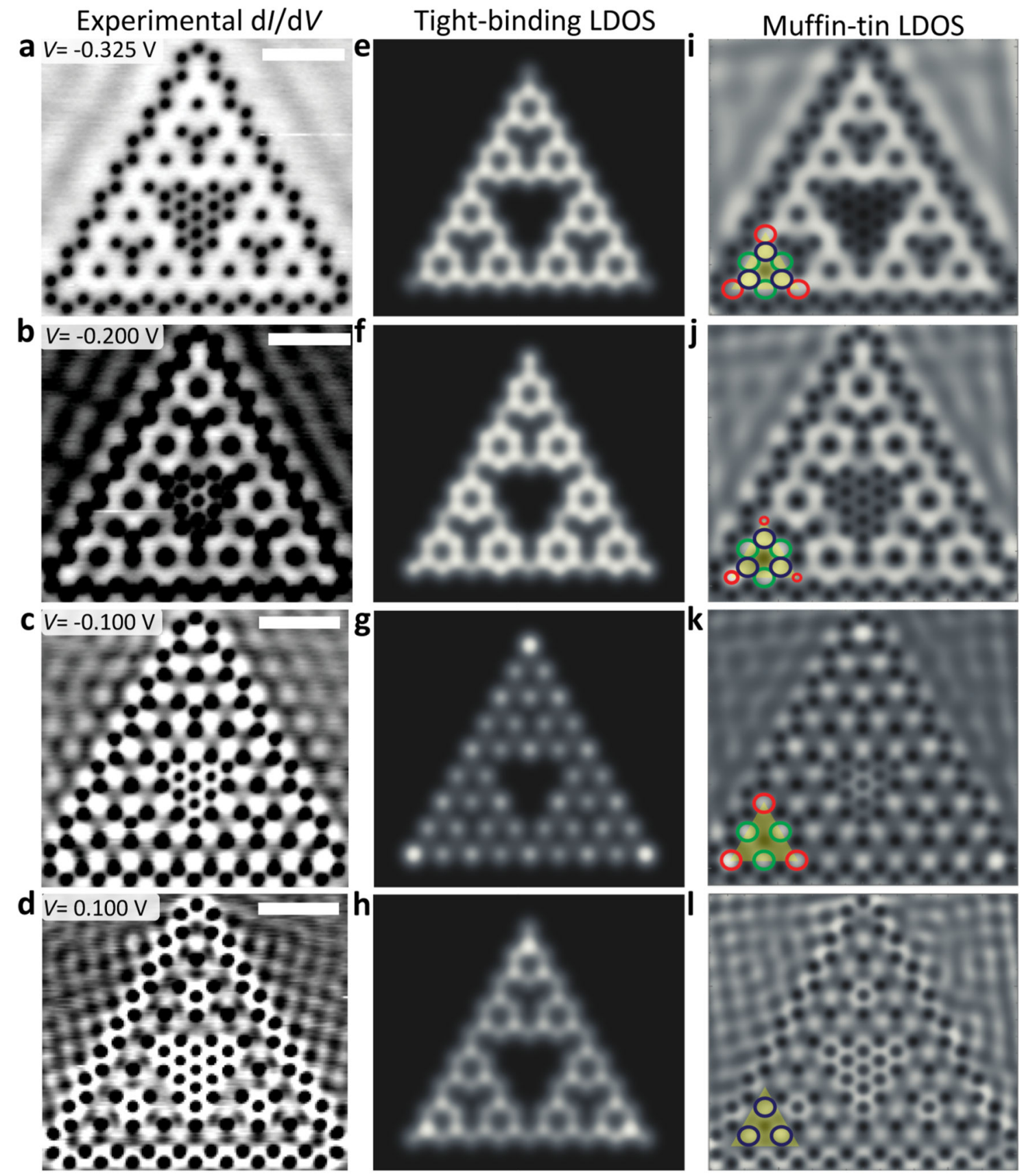

Figure 2. Wave-function mapping.

a-d, Differential conductance maps acquired above a $G(3)$ Sierpiński triangle at bias voltages $-0.325 \mathrm{~V},-0.200 \mathrm{~V},-0.100 \mathrm{~V}$, and $+0.100 \mathrm{~V}$. Scale bar: $5 \mathrm{~nm}$. e-h, LDOS maps at these energies calculated using the tight-binding model. i-l, LDOS maps simulated using the muffin-tin approximation. As a guide to the eye, a $G(1)$ building block is indicated, in which a larger radius of the circles corresponds to a larger LDOS at an atomic site, while no circle indicates a node in the LDOS. 

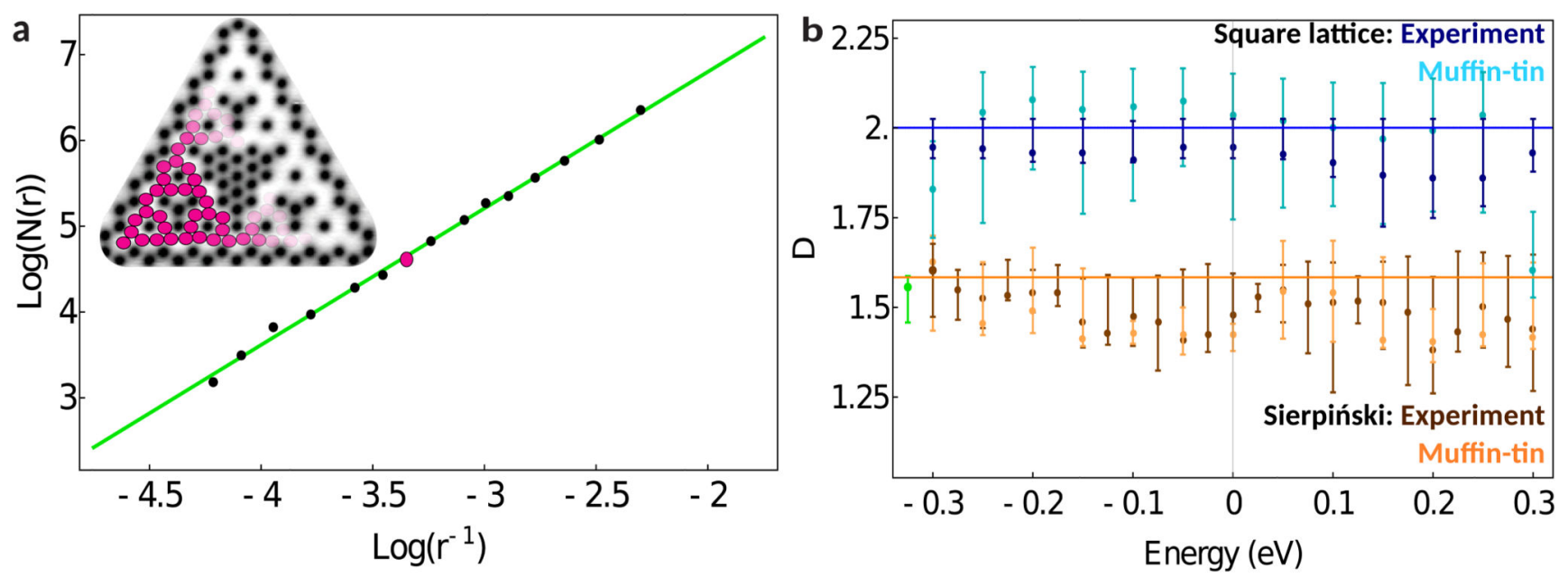

Figure 3. Fractal dimension of the Sierpiński wave-function maps.

a, The box-counting dimension of the wave-function map acquired at $V=-0.325 \mathrm{~V}$ is obtained from the slope of $\log (N)$ vs. $\log \left(r^{-1}\right)$. The magenta dot indicates the radius $r$ of the $N$ circles used in the inset. Inset: Schematic of the box-counting method, where $N$ circles with radius $r$ cover the contributing experimental LDOS above the threshold of $45 \%$ at $V=$ $-0.325 \mathrm{~V}$ (see Supplementary Information for the determination of this threshold). $\mathbf{b}$, Determination of the fractal dimensions of the LDOS of the $G(3)$ Sierpiński triangle (orange) and comparison with the 2D square lattice from Ref. 3 (blue) for the experimental (dark) and muffin-tin (light) wave function maps. The solid lines indicate the geometric Sierpiński Hausdorff dimension $(D=1.58)$ and that of the square lattice $(D=2)$. The error bars display the maximum of the error in determining the fractal dimension at different LDOS thresholds, which is between $45 \%$ - 65\% (60\% - 90\%) for experiment (muffin-tin)) and the error in determining the slope of the loglog plot as seen in $\mathbf{a}$. The green result at $-0.325 \mathrm{~V}$ is obtained from the slope in $\mathbf{a}$. The fluctuations in the dimension are caused by nodes in the LDOS maps at different energies. 


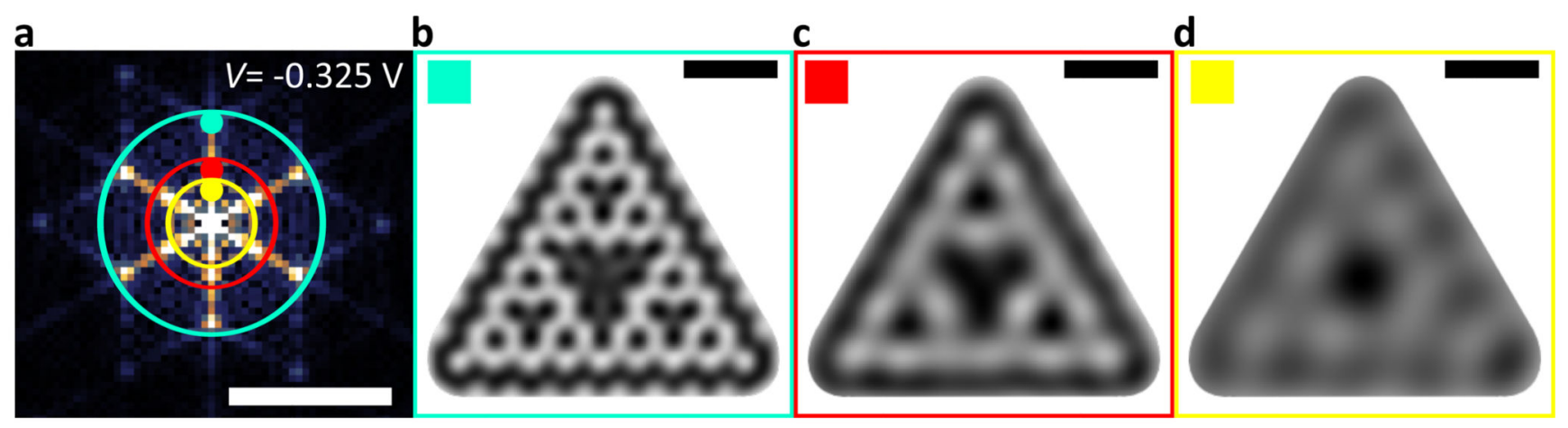

Figure 4. Fourier analysis of wave-function maps.

a, Fourier transform of the experimental differential conductance map at $-0.325 \mathrm{~V}$. The $k$ values outside the circles are excluded from the Fourier-filtered images in b-d. Scale bar: $k=$ $3 \mathrm{~nm}^{-1}$. b-d, Wave-function map at $-0.325 \mathrm{~V}$ after Fourier-filtering, including merely the $k$ values within the turquoise (b), red (c), and yellow (d) circles indicated in a. Scale bar: 5 nm. 